\title{
Non-Virilizing Congenital Adrenal Hyperplasia in a Female Patient with a Novel HSD3B2 Mutation
}

\author{
Ursina Probst-Scheidegger ${ }^{a, b}$ Sameer S. Udhane ${ }^{c}$ Dagmar l'Allemand ${ }^{b}$ \\ Christa E. Flück ${ }^{c}$ Núria Camats ${ }^{c, d}$ \\ a Pediatric Department, Kantonsspital Winterthur, Winterthur, ${ }^{b}$ Children's Hospital of Eastern Switzerland, St. Gallen, \\ and 'Pediatric Endocrinology and Diabetology, Department of Pediatrics and Department of Clinical Research, \\ University Children's Hospital Bern, Bern, Switzerland; d Growth and Development Research Unit, Vall d'Hebron \\ Research Institute (VHIR), Center for Biomedical Research on Rare Diseases (CIBERER), Instituto de Salud Carlos III, \\ Barcelona, Spain
}

\section{Key Words}

$3 \beta$-Hydroxysteroid dehydrogenase $\cdot$ Congenital adrenal hyperplasia $\cdot H S D 3 B 2 \cdot$ Newborn screening

\begin{abstract}
Classic $3 \beta$-hydroxysteroid dehydrogenase type 2 (3ß-HSD II) deficiency causes congenital adrenal hyperplasia with glucocorticoid, mineralocorticoid, and sex steroid deficiency. We present a female patient with congenital adrenal hyperplasia detected in newborn screening due to elevated $170 \mathrm{H}-$ progesterone. Female external genitalia and non-measurable androgen levels elicited the suspicion of a defect early in the steroid cascade. Two loss-of-function HSD3B2 mutations ( 1 novel) were detected and confirmed in silico. We argue that in a girl with glucocorticoid and mineralocorticoid deficiency without virilization, $3 \beta-H S D$ II deficiency is an important differential diagnosis. $170 \mathrm{H}$-progesterone may initially be elevated due to placental and peripheral activity of $3 \beta$-HSD I, whereas dehydroepiandrosterone may not be increased.

(c) 2016 S. Karger AG, Basel
\end{abstract}

(2) 2016 S. Karger AG, Basel

$1661-5425 / 16 / 0104-0200 \$ 39.50 / 0$
$3 \beta$-Hydroxysteroid dehydrogenase ( $3 \beta$-HSD) is a key enzyme in steroidogenesis, responsible for the conversion of $\Delta 5$ - to $\Delta 4$-steroids. Thus, it converts the $\Delta 5$-steroids pregnenolone, 17OH-pregnenolone, dehydroepiandrosterone (DHEA), and androstenediol to the $\Delta 4$-steroids progesterone, $17 \mathrm{OH}$-progesterone (17OHP), androstenedione, and testosterone, respectively [Simard et al., 1995]. In humans, the enzyme exists in 2 isoforms, type 1 being expressed in the placenta and peripheral tissues, and type 2 in adrenals and gonads [Simard et al., 1995].

Deficiency in $3 \beta$-HSD type 2 leads to a rare form of congenital adrenal hyperplasia (CAH). Complete loss of function of the enzyme is responsible for adrenal crisis in the newborn, including hypocortisolism and salt loss. Genital development is impaired in males, ranging from hypospadias to $46, \mathrm{XY}$ disorder of sex development with nearly female appearing external genitalia [Simard et al., 2005]. Females with $3 \beta$-HSD type 2 ( $3 \beta$-HSD II) deficiency present with no or mild virilization by means of elevated $\Delta 5$ steroids that are peripherally converted to androgens by activity of the type 1 isoenzyme of $3 \beta$-HSD [Simard et al.,

\section{KARGER}

E-Mail karger@karger.com www.karger.com/sxd
Núria Camats

Growth and Development Research Unit, VHIR

Hospital Universitari Vall d'Hebron, Passeig Vall d'Hebron, 119-129 ES-08035 Barcelona (Spain)

E-Mail nuria.camats@ vhir.org 
Table 1. Biochemical values of the patient at different ages

\begin{tabular}{|c|c|c|c|c|c|c|c|c|}
\hline $\mathrm{K}, \mathrm{mmol} / \mathrm{l}$ & & 5.8 & $6.1 \uparrow$ & & & & & \\
\hline 17OHP, nmol/l & $244 \uparrow$ & $124 \uparrow$ & & $32.7 \uparrow$ & $26.9 \uparrow$ & $<0.21$ & 3.8 & 5.0 \\
\hline DHEA-S, $\mu \mathrm{mol} / \mathrm{l}$ & & 12 & & & & $<0.41$ & $<0.41$ & $<0.41$ \\
\hline $\mathrm{D}^{4} \mathrm{~A}, \mathrm{nmol} / \mathrm{l}$ & & & & & & $<1.05$ & $<1.05$ & $<1.05$ \\
\hline ACTH, ng/l & & $549 \uparrow$ & & & & & & $490 \uparrow$ \\
\hline Cortisol, nmol/l & & $92 \downarrow$ & & & & & & $<28 \downarrow$ \\
\hline Renin, pg/ml & & 116 & & & & & & $1,414 \uparrow$ \\
\hline Aldo, $\mathrm{pmol} / \mathrm{l}$ & & 1804 & & & & & & $93 \downarrow$ \\
\hline
\end{tabular}

Arrows indicate higher $(\uparrow)$ or lower $(\downarrow)$ values. NBS = Newborn screening; 11-DOC = 11-deoxycortisol; DHEA-S = dehydroepiandrosterone sulfate; $\mathrm{D}^{4} \mathrm{~A}=$ androstenedione; $\mathrm{ACTH}=$ adrenocorticotropic hormone; Aldo = aldosterone; $\rightarrow=$ first dose.

1995, 2005]. The typical presentation of the non-salt-losing forms is premature pubarche in both sexes.

The molecular etiology of $3 \beta$-HSD deficiency lies in a defect in the HSD3B2 gene, coding for the isoform II of the enzyme (type 2) [Rheaume et al., 1995].

\section{Case Report}

A healthy full-term female neonate, born to a non-consanguineous couple from Eastern Switzerland, was admitted to our pediatric endocrine service on day 8 of life for further evaluation of increased 17OHP in newborn screening. She presented in no physical distress, feeding well and gaining weight appropriately.

On clinical examination, we found normal female external genitalia without virilization or palpable gonads. Ultrasound revealed female internal genitalia with a slightly stimulated uterus and normal ovaries with few follicles. Biochemical analyses revealed increased adrenocorticotropic hormone with low cortisol, increased 17OHP, normal DHEA, 11-deoxycortisol, and increased renin (table 1).

For suspected CAH with glucocorticoid deficiency, the patient was started on hydrocortisone (HC) immediately. The following day, she developed salt loss ( $\mathrm{Na} 129 \mathrm{mmol} / \mathrm{l}, \mathrm{K} 6.1 \mathrm{mmol} / \mathrm{l}$ ) and was therefore also started on fludrocortisone (FC) and $\mathrm{NaCl}$ supplements (table 1). On continuous HC and FC replacement, psychomotor and physical development was normal.

At 7 months of age, the patient was admitted to the hospital for further evaluation: $\mathrm{HC}$ and FC were discontinued. Serum steroids were measured $36 \mathrm{~h}$ after the last dose of HC and FC. Results confirmed glucocorticoid and mineralocorticoid deficiency (table 1). However, there was no increase in 17OHP or androgens. Due to the severe lack of all steroid hormones, we suspected a defect high up in the steroid cascade.

Non-Virilizing $\mathrm{CAH}$ in a Female Patient with a HSD3B2 Mutation

\section{Results}

\section{Genetic Analysis}

Genetic analysis revealed that the patient was compound heterozygous for 2 mutations in the HSD3B2 gene (fig. 1A). One mutation (c.503delC) caused a frameshift leading to a premature stop codon (A168Vfs*6). The other was a nonsense (stop) mutation shortly after the first one (c.512G $>\mathrm{A} ; \mathrm{W} 171 \mathrm{X})$. The combination of both mutations was considered to cause the phenotype of the patient.

Both mutations lead to a shorter and aberrant gene product. The normal peptide product is 285 amino acids long, whereas the frameshift A168Vfs*6 mutation coded for a peptide of 172 amino acids and the stop W171X mutation for a 170 amino acid peptide (fig. 1B). The parents were heterozygous for the 2 mutations (fig. 1A). Two years later, a brother was born with normal male external genitalia and a normal urinary steroid profile at birth. The genetic analysis showed that he was heterozygous for the A168Vfs*6 paternal mutation (fig. 1A).

\section{In silico Analysis}

The 2 mutations were further analyzed in silico. We analyzed the human HSD3B2 peptide residues 167-173 involved in the mutations by sequence alignment (fig. 2) and structural modeling (fig. 3 ). The 2 mutations were checked by comparing the positions of these amino acids in different mammalian HSD3B sequences (fig. 2A). 
Fig. 1. Genetic analysis of the HSD $3 B 2$ gene. A Two mutations in the HSD3B2 gene of the patient were detected. One was a 1-bp deletion responsible for a shift of the reading frame causing 5 amino acid changes followed by a stop codon (c.503delC; A168Vfs*6). The other mutation, only 9 nucleotides downstream, was a 1-bp change which produced a stop codon (c.512G>A; W171X). The parents presented 1 mutation each and the younger brother the paternal mutation. The HSD $3 B 2$ gene was amplified (exonic coding sequences including intronic boundaries) using specific primers and PCR amplification protocols as described elsewhere. Obtained sequences were compared to NG_013349.1 (http://www.ncbi.nlm.nih. gov/). B The HSD3B2 gene alleles of the patient code for 2 different peptide sequences, both shorter than the wild type.

Fig. 2. Partial sequence alignment and secondary structure prediction of the HSD3B2 protein. A Human HSD3B2 was partially aligned with homologous sequences from 6 mammalian species. Position A168 is conserved among different species, whereas position W171 is replaced with S171 in mouse and rat. The alignment of the HSD3B2 amino acid sequence (P26439) was adapted from the UniProt database, and the other mammalian sequences were also available in the same database (www.uniprot.org). Human: Homo sapiens; Canfa: dog, Canis familiaris; Pig: Sus scrofa; Bovine: cattle, Bos taurus; Mouse: Mus musculus; Rat: Rattus norvegicus; Macmu: Rhesus macaque, $\mathrm{Ma}$ caca mulatta. B Prediction of the secondary structure of the HSD3B2 protein. The positions of A168Vfs*6 and W171X and previously reported mutations are highlighted. All these mutations are close to the alpha helix and coil region predicted with the confidence value (depicted in line 3). W171 and L173 are predicted to be located in a disordered region, indicated by question marks. Prediction of the secondary structure was obtained from the Phyre2 server [Kelley and Sternberg, 2009]. The first line indicates the amino acid sequence and the second line the secondary structure prediction.

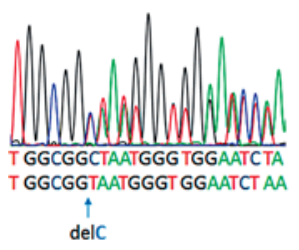

c.503delC: A168Vfs*6

A
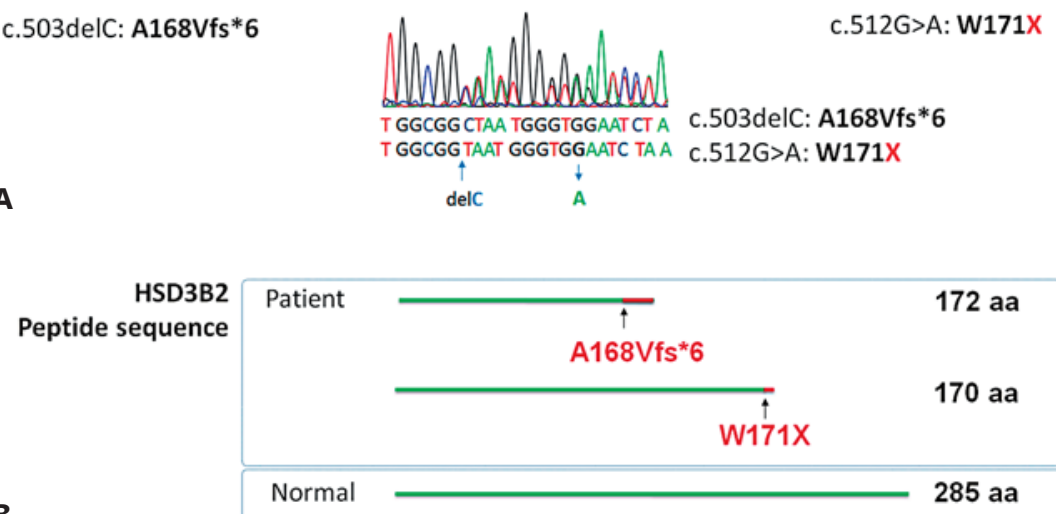

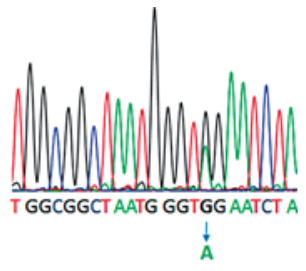

$.512 G>A:$ W171X

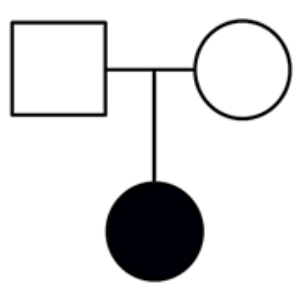

T GGCGG CTAA TGGGTGGAATCT A c.503delC: A168Vfs*6 $\begin{array}{ll}\uparrow & \text { delc }\end{array}$
B

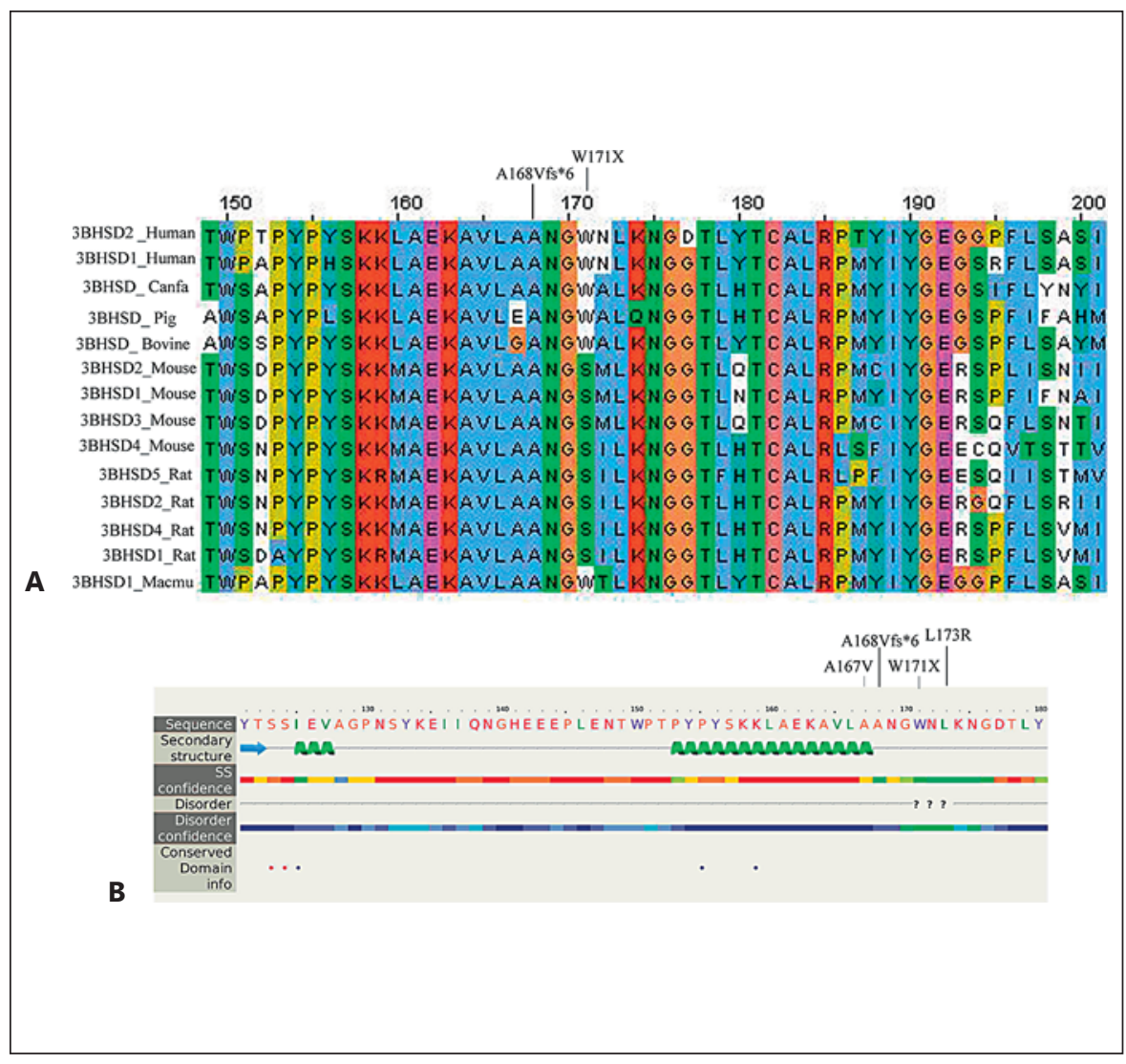




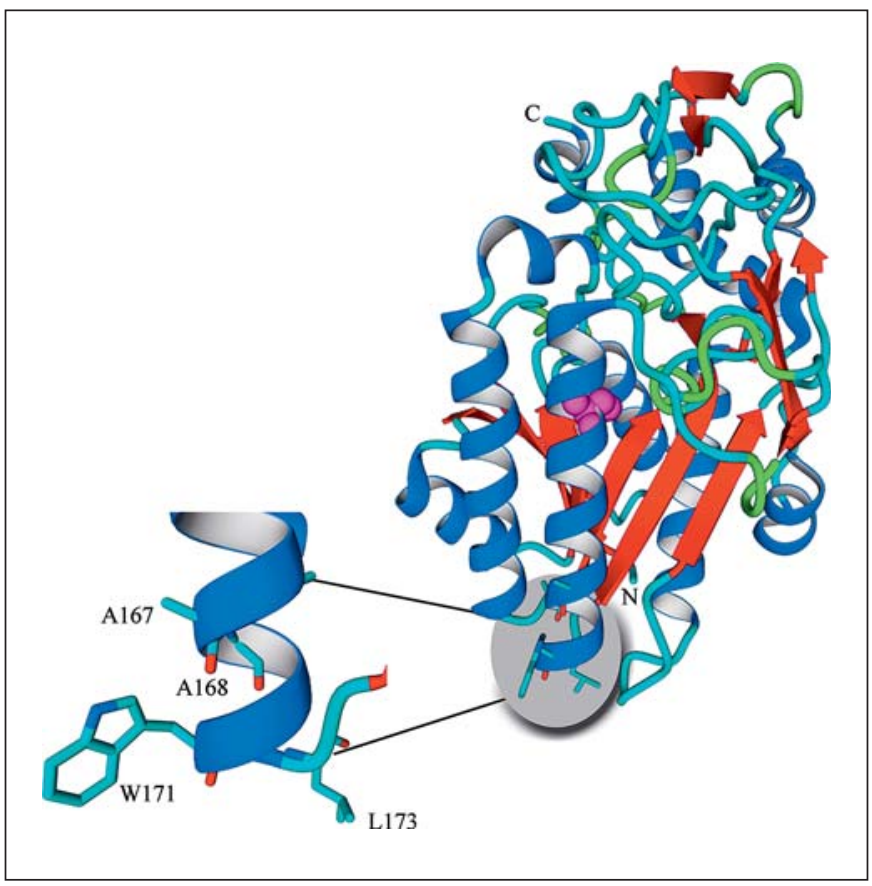

Fig. 3. Structure of HSD3B2. The positions of A168Vfs*6 and W171X and the previously reported mutations A167V and L173R (circled and magnified) are highlighted in the 3D model structure of the HSD2B2 protein. The N-terminal (N) and C-terminal (C) ends are indicated, and the amino acid region from 167 to 173 is encircled and shown in a larger resolution. The position of amino acid 158 for NAD-ligand binding sites is shown in the central ball shape (based on the UniProt database). The 3D model structure was obtained using the HSD3B2 protein (NP_000189.1, P26439) sequence. We obtained the HSD3B2 structural model (based on clz45A) from the Phyre2 server [Kelley and Sternberg, 2009], which was visualized by the YASARA program (www.yasara.org).

Amino acid A168 is well conserved across all the species, but tryptophan at position 171 is substituted with serine in mouse and rat species (fig. 2A). The structural studies showed that amino acids 167-173 are located in the alpha helix and coil region of the protein and are close to the active site and NAD-binding site of the HSD3B2 protein (fig. 3). We suggest that the A168Vfs*6 and W171X mutations lead to truncated HSD3B2 proteins without enzymatic activity. Other previously reported mutations, A167V and L173R, are located in the vicinity of our patient's mutations (fig. 3), A167V not having a major effect on the native structure of HSD3B2 [Nayak et al., 1998; Moisan et al., 1999], whereas heterozygous L173R reduced 3 $\beta$-HSD enzyme activity by half [Russell et al., 1994; Moisan et al., 1999]. Our analysis predicted that L173R is located in a disordered region of the protein (fig. 2B).

\section{Discussion}

We describe a patient with a novel HSD3B2 mutation (A168Vfs*6) and a previously reported one (W171X). The latter (maternal mutation) was described 20 years ago in 2 families also from Eastern Switzerland [Rheaume et al., 1992]. We were able to track medical records and suspect a familial relation. Patients in these families, who carried the mutation in a homozygous fashion, presented with a classic form of $3 \beta-H S D$ deficiency with severe salt wasting.

Our female patient was diagnosed with severe glucocorticoid and mineralocorticoid deficiency in newborn screening due to elevated 17OHP. The lack of androgens as well as the completely normal female genitalia made us suspect a defect high up in the steroid cascade. By molecular analysis, our patient could be diagnosed with a compound heterozygous HSD $3 B 2$ defect. The increase in $17 \mathrm{OHP}$ could be interpreted as a product of placental activity of the isoform I of $3 \beta-H S D$. Surprisingly, DHEA was not elevated at birth or at 7 months after discontinuation of treatment, probably due to the remaining activity of $3 \beta$-HSD I or to the conversion in the periphery also of $3 \beta$-HSD I. We expect an increase in DHEA during adrenarche with otherwise absent increase in steroid hormones and lack of spontaneous pubertal development.

\section{Conclusion}

In a female $\mathrm{CAH}$ patient without virilization, $3 \beta-\mathrm{HSD}$ II deficiency must be considered. 17OHP can be elevated in the first few days of life due to placental and peripheral $3 \beta$-HSD I activity. DHEA is not necessarily increased.

We report a novel HSD3B2 mutation, c.503delC, clinically causing a severe impairment of $3 \beta$-HSD II activity and leading to a nearly complete deficiency in glucocorticoids, mineralocorticoids, and sex steroids in our phenotypically non-virilized female $\mathrm{CAH}$ patient.

\section{Acknowledgments}

We thank the presented patient and her family as well as Dr. Jana Malikova for her help in this study. 


\section{Statement of Ethics}

Oral informed consent was given by the patient's parents.

\section{Disclosure Statement}

The authors have no conflicts of interest to declare.

\section{References}

Kelley LA, Sternberg MJ: Protein structure prediction on the Web: a case study using the Phyre server. Nat Protoc 4:363-371 (2009).

-Moisan AM, Ricketts ML, Tardy V, Desrochers M, Mebarki F, et al: New insight into the molecular basis of 3beta-hydroxysteroid dehydrogenase deficiency: identification of eight mutations in the HSD3B2 gene eleven patients from seven new families and comparison of the functional properties of twenty-five mutant enzymes. J Clin Endocrinol Metab 84: 4410-4425 (1999).
Nayak S, Lee PA, Witchel SF: Variants of the type II 3beta-hydroxysteroid dehydrogenase gene in children with premature pubic hair and hyperandrogenic adolescents. Mol Genet Metab 64:184-192 (1998).

Rheaume E, Simard J, Morel Y, Mebarki F, Zachmann M, et al: Congenital adrenal hyperplasia due to point mutations in the type II 3 betahydroxysteroid dehydrogenase gene. Nat Genet 1:239-245 (1992).

Rheaume E, Sanchez R, Mebarki F, Gagnon E, Carel JC, et al: Identification and characterization of the G15D mutation found in a male patient with 3 beta-hydroxysteroid dehydrogenase ( 3 beta-HSD) deficiency: alteration of the putative NAD-binding domain of type II 3 beta-HSD. Biochemistry 34:2893-2900 (1995).
Russell AJ, Wallace AM, Forest MG, Donaldson MD, Edwards CR, Sutcliffe RG: Mutation in the human gene for 3 beta-hydroxysteroid dehydrogenase type II leading to male pseudohermaphroditism without salt loss. J Mol Endocrinol 12:225-237 (1994).

Simard J, Sanchez R, Durocher F, Rheaume E, Turgeon C, et al: Structure-function relationships and molecular genetics of the 3 beta-hydroxysteroid dehydrogenase gene family. J Steroid Biochem Mol Biol 55:489-505 (1995).

Simard J, Ricketts ML, Gingras S, Soucy P, Feltus FA, Melner MH: Molecular biology of the 3beta-hydroxysteroid dehydrogenase/delta5delta4 isomerase gene family. Endocr Rev 26: 525-582 (2005). 